

Michigan Technological University Digital Commons @ Michigan Tech

9-1-1997

\title{
Fluctuation properties of precipitation. Part I: On deviations of single-size drop counts from the Poisson distribution
}

Alexander Kostinski

Michigan Technological University

A. R. Jameson

RJH Scientific, Inc.

Follow this and additional works at: https://digitalcommons.mtu.edu/physics-fp

Part of the Physics Commons

\section{Recommended Citation}

Kostinski, A., \& Jameson, A. R. (1997). Fluctuation properties of precipitation. Part I: On deviations of single-size drop counts from the Poisson distribution. Journal of the Atmospheric Sciences, 54(17), 2174-2186. http://dx.doi.org/10.1175/1520-0469(1997)054<2174:FPOPPI>2.0.C0;2

Retrieved from: https://digitalcommons.mtu.edu/physics-fp/254

Follow this and additional works at: https://digitalcommons.mtu.edu/physics-fp

Part of the Physics Commons 


\title{
Fluctuation Properties of Precipitation. Part I: On Deviations of Single-Size Drop Counts from the Poisson Distribution
}

\author{
A. B. Kostinski \\ Department of Physics, Michigan Technological University, Houghton, Michigan
}

A. R. JAMESON

RJH Scientific, Inc., Alexandria, Virginia

(Manuscript received 13 September 1996, in final form 29 January 1997)

\begin{abstract}
The traditional statistical description of the spatial and temporal distributions of cloud droplets and raindrops is the Poisson process, which tends to place the drops as uniformly as randomness allows. Yet, the "clumpy" nature of clouds and precipitation is apparent to most casual observers and well known to cloud physicists. Is such clumpiness consistent with the Poisson statistics? The authors explore the possibility of deviations from the Poisson distribution using temporal raindrop counting experiments. Disdrometer measurements during the passage of a squall line strongly indicate that a mixture of Poisson distributions (Poisson mixture) provides a better description of the frequency of drop arrivals per unit time in variable rain than does a simple Poisson model. Poisson mixture generally yields distributions different from Poissonian. While the validity of the Poisson mixture model to smaller scales requires much finer temporal resolution than available in this study, these results do show that one must carefully interpret the statistical and physical meaning of average drop concentrations when the measurements are collected through variable rain, whether observed by airborne or ground-based instruments. Statistically, the variance in the measurements is greatly increased, due to the added variability from the rain field, thus minimizing the reduction of the variance normally achieved by increasing the sample mean $(N)$. In fact, in some cases the variance of relevant distributions scales as $N^{2}$ rather than $N$, thereby making the relative fluctuations independent of $N$. Consequently, the sampling criteria proposed by Cornford are not necessarily generally applicable. Moreover, the authors conjecture that in most clouds the distribution of drop concentrations in small volumes may be more aptly described by a Poisson mixture rather than by a pure Poisson distribution. This may have significant implications with regard to the droplet growth and the evolution of rain.
\end{abstract}

\section{Introduction}

The soothing sound produced by raindrops striking the roof of a house has long been considered a classic example of a Poisson process, that is, the probability of $k$ drops arriving per unit time on a surface is described by the Poisson distribution (e.g., van Kampen 1992, 34). However, closer attention at times reveals hints of a rhythm in the rain. Presumably such pulsations have to do with "clumpiness" or "patchiness" often observed during a storm. While the structure of rain has been explored using radars (e.g., Crane 1990), such analyses have been restricted to spatial scales larger than about $1 \mathrm{~km}$.

On scales much smaller than $1 \mathrm{~km}$, it is usually assumed that raindrops and cloud droplets are nearly

Corresponding author address: Prof. Alexander B. Kostinski, Department of Physics, Michigan Technological University, 1400 Townsend Drive, Houghton, MI 49931.

E-mail: kostinsk@mtu.edu evenly distributed in space to the extent allowed by randomness. We quote from Rogers and Yau (1989, 134):

Even in a well-mixed cloud with the same average droplet concentration throughout, there will be local variations in concentration. In particular, if $n$ denotes the average concentration of droplets in a given size interval, then the number $m$ of such droplets in a volume $V$ obeys the Poisson probability law .... .

Nevertheless, measurements in clouds often reveal complex structures in drop concentrations and cloud water contents. So what exactly, then, does "well mixed" mean?

To the extent that turbulence is responsible for stirring, clumpiness is to be expected because turbulence is well known to be intermittent and spotty. That is, an initially homogeneous "blob" of cloud droplets (assumed sufficiently light to be regarded as passive tracers) will be twisted and distorted by a succession of turbulent eddies as time progresses. The resultant 
clumpy "structures" must be viewed stochastically. One might, then, expect fluctuations in particle count to be in excess of that implied by Poisson statistics because of local variations in the mean concentrations, that is, because of clumpiness. (Here the word "local" refers to scales shorter than a typical clump size.) Although perhaps less readily visualized for precipitation, raindrops will often experience a spectrum of air velocity fluctuations, as well as drop interactions and differential advection, likely to produce clustering similar to that in clouds. With regard to raindrops, a seminal work appears to be that of Cornford (1967). By assuming that the randomness obeys the Poisson distribution, he was able to define precisely when a mean concentration corresponding to a particular drop size had been adequately sampled. Since then, for both clouds and rain, it is generally accepted that Poisson statistics apply (see also Gillespie 1972, 1975; Young 1993).

Yet the fine but visible "streakiness" of rain falling from clouds or striking the pavement during a thunderstorm is a matter of common experience. That is, over brief periods the spatial distribution of the raindrops no longer appears even but clumpy, with clusters of raindrops in close proximity separated by extended regions containing few if any drops as can be clearly seen in the so-called sheets of rain passing across the pavement during thunderstorms, for example.

A description of such randomness is important when attempting to measure drop concentrations, for example, since it is necessary to decide when the mean concentration at a particular drop size has been adequately sampled (Cornford 1967). In addition, the spatial distribution of raindrops and cloud droplets is relevant to the evolution of rain, particularly with regard to stochastic collection models (see discussion in Pruppacher and Klett 1978). ${ }^{1}$

We propose here to test the following question: Does the distribution of raindrop counts deviate from the Poissonian one? To explore this, we will use the property that the mean and variance are equal for a Poisson distribution. In contrast, as shown below, the variance can be greatly enhanced for a mixture of Poisson distributions because of the additional uncertainty introduced by the randomly varying local mean. Our question can then be rephrased to become: Does the variance of raindrop counts exceed the Pois-

\footnotetext{
${ }^{1}$ A particularly vexing problem in cloud physics has been to explain the rapid broadening of drop size spectra and appearance of the first raindrops. Theory suggests much slower development than is actually observed. Among the proposed solutions is the notion of "ultra-giant nuclei" (Johnson 1982). This concept appears quite reasonable and replaces the earlier notion of "statistically fortunate" drops (Twomey 1964). Using arguments based upon Poissonian statistics, this latter explanation has been discounted (Gillespie 1972, 1975; Young 1993). Nevertheless, if the variances of concentration fluctuations are enhanced as in a Poisson mixture, then the original argument of Twomey may still have some validity.
}

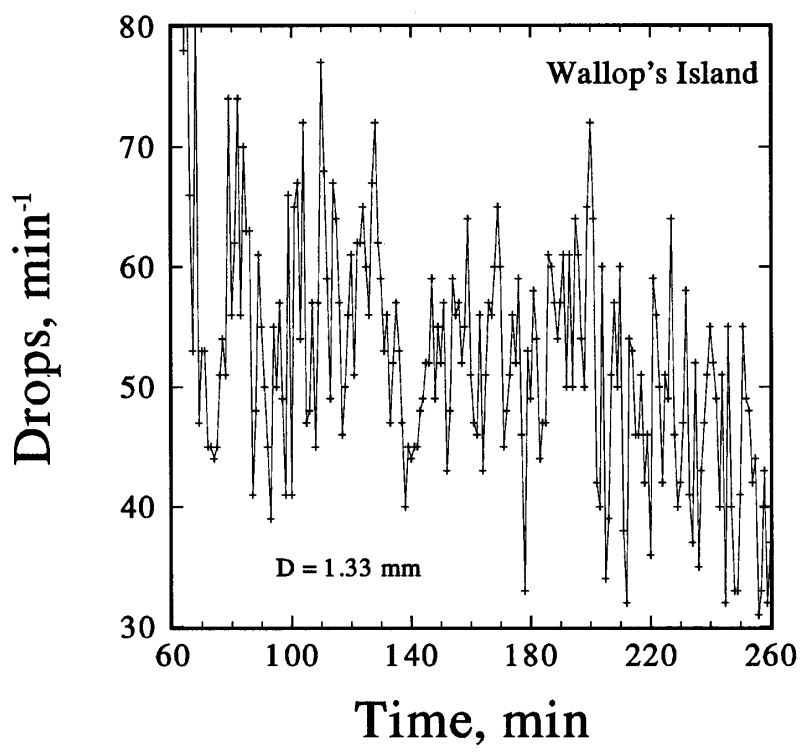

FIG. 1. Time series of the number of drops per minute in the convective, stratiform rain at Wallop's Island, Virginia.

son value? To find out, we will count raindrops as a function of time and have the data tell us whether or not the distribution is Poissonian.

In time, the assumptions underlying the Poisson distribution (e.g., Feller 1968, 157-159) are that the random process is stationary, that two events are much less likely than one or zero on sufficiently short timescales, and that events in nonoverlapping time intervals are statistically independent. With regard to space, the assumptions are that the probability of having more than one occurrence in sufficiently small volumes is negligible compared to having none or one and that the occurrences in nonoverlapping regions are statistically independent. One can question these assumptions since rain evolves through the processes of drop collision, breakup, and coalescence, leading to possible correlations among the drops (Bayewitz et al. 1974).

The physics of this paper is in the conjecture that in both, time and space, the uneven and intermittent mixing by atmospheric turbulence, among other factors, causes clumpiness of clouds and rain on the scales of most counting experiments. This clumpiness, in turn, is associated with correlations between drop counts corresponding to nonoverlapping time (or space) intervals. Such correlations represent departures from the statistical independence assumption and, therefore, lead to deviations from the Poisson distribution. In fact, these correlations define what we mean by "size" (temporally or spatially) of the clumps or patches of rain as determined by the appropriate coherence times.

In this study, the fundamental data are the one minute disdrometer counts of the number of drops of a given size as illustrated in Fig. 1. We regard such counts and 
their time series as realizations of a random variable and random process, respectively. Indeed, an observed time series of counts (with a single disdrometer) is but one "realization" of an infinite number of such series. While this "ensemble" of time series can be regarded as stationary or even ergodic, each realization is an unpredictable expression of time-dependent "variable meteorological conditions.", 2

In particular, we assume that each 1-min count is drawn from a larger "patch" of rain characterized by some mean number of drops per minute. We incorporate the concept of rain patchiness on scales characterized by some coherence time (say, $10 \mathrm{~min}$ ). More precisely, since the 1-min measurements are much shorter than the 10-min timescale, one can argue that each 1-min measurement is one realization of a Poisson process with a fixed local mean associated with a particular patch. As different rain patches pass over a detector in an unknown and unpredictable (and therefore random) manner, the local mean itself fluctuates, leading to a Poisson mixture and increased variance. Throughout this paper, the words local or instantaneous will refer to measurement durations shorter than corresponding coherence times.

We report in this work that the temporal distribution of raindrops appears to be non-Poissonian at least over the long timescales of these observations. Moreover, we will see that such statistics are appropriately described in terms of a probability mixture of Poisson distributions. This finding is not just an intellectual curiosity. For example, the validity of a measured drop size distribution depends upon proper sampling, as pointed out by Cornford. Thus, depending upon the length of the observation period, sampling time, and the coherence time (patch size), the Cornford criteria may not always apply, particularly, if ever increasingly finer resolution counts lead to ever decreasing coherence times. Furthermore, on longer timescales, the physical interpretation of a "mean concentration" itself becomes obscured by the variability (increased variance) of the rain. This is so because a mixture of Poisson distributions is, in general, not Poisson distributed. In fact, as we shall see, its variance no longer scales as $N$ (the average number of drops), but rather as $N^{2}$, which prevents convergence of the relative counting error with progressively larger numbers of sampled drops.

Furthermore, although this analysis is based upon temporal measurements, there are reasons to believe that such variability also occurs spatially as aircraft measurements abundantly suggest. If so, it is reason-

\footnotetext{
${ }^{2}$ Here it should be noted that small-scale inhomogeneities or clumpiness in clouds and rain do not preclude statistical homogeneity. The probability distribution can still be statistically homogeneous because its moments, for example, mean and variance are unaffected by shifts in the choice of origin (either in time or space).
}

able to expect that there may also be a "super-Poissonian" variance in the spatial distribution of raindrops caused by clumpiness and clustering. If true, this may be important to our understanding of the evolution of rain, to the scattering of microwaves by raindrops, and even to cloud and precipitation evolution. For example, any additional clustering associated with the deviations from the Poisson statistics may not only affect the scatter and absorption of solar and terrestrial radiation but may also lead to a more rapid growth of a few "favored" cloud droplets into the first raindrops, a critical step for the subsequent conversion of cloud into rainwater. ${ }^{3}$

In the next section we consider drops of constant size and begin by presenting classic Poisson statistics applicable to evenly mixed conditions when the mean concentration is a constant in time and space. We then proceed to the more general case when the local mean concentration varies. Finally, these theoretical results are used to interpret measurements of nearly uniform size in actual rain.

\section{Theory}

The Poisson distribution is often derived as a limiting case of the binomial one. This perspective, however, tends to obscure the reasons for the pivotal place of the Poisson distribution in describing counting processes of physical sciences. The role of the assumption of statistical independence is of particular importance to us here. The general conditions underlying the Poisson distribution are that (e.g., Goodman 1985)

1) the probability of a single event occurring during the interval $t, t+\delta t$ is proportional to $\delta t$;

2) the probability of more than one event in the interval $t, t+\delta t$ is vanishingly small for sufficiently small $\delta t$; and

3) event counts in nonoverlapping time intervals are statistically independent.

If these conditions are satisfied, the Poisson distribution results and is given by (e.g., Feller 1968)

$$
p(k)=\frac{(\bar{k})^{k} \exp (-\bar{k})}{k !},
$$

where $\bar{k}$ is the mean value and $k$ is the random variable (number of events). With respect to raindrops, there are two interpretations of $\bar{k}$ and $k$. In time, $\bar{k}$ can be

\footnotetext{
${ }^{3}$ To illustrate this point, we quote from Rogers and Yau (1989, 134): "Some statistically 'fortunate' drops fall through regions of locally high droplet concentration, experiencing more than the average number of collisions early in their development, and are subsequently in a favored position to continue to grow relatively rapidly." Note that the super-Poissonian fluctuations in the number of collisions can significantly accelerate the fortunate droplet growth.
} 
considered the mean number of drops per unit time for the entire series of trials, while $k$ is then the random number actually observed during one particular counting interval of duration $t$ (one trial). In space, $\bar{k}$ can be interpreted as the mean concentration of drops per unit volume, while $k$ would then be the number of drops actually observed in one unit volume. For such a distribution the mean and variance are equal $\left(\bar{k}=\sigma_{k}^{2}\right)$, where $\sigma^{2}$ is the variance (e.g., Wilks 1995). Thus, the relative fluctuation $\left(\sigma_{k} / \bar{k}\right) \mathrm{de}-$ creases with increasing $\bar{k}$ as $1 / \sqrt{\bar{k}}$ that is, the more drops sampled, the better.

However, if the mean concentration $(\bar{k})$ is an unpredictable, random variable in time and space, this Poissonian "evenly mixed" model does not apply. Variability in $\bar{k}$ might occur because of differential advection among different patches having different $\bar{k}$ or because of horizontal gradients in the vertical wind shear, for example. Also, drops of many different sizes may accidentally be nearly collocated. Because each drop size has a different mean concentration, clustering results. We postpone such considerations and confine ourselves here to the simpler scenario of drops of one size. The question, then, is how to describe the statistics when conditions (i.e., $\bar{k}$ ) are variable?

Let us regard a given time series of drop counts as one member of an ergodic ensemble of such series. This is akin to asserting that the time series is in some sense "typical" so that another disdrometer nearby sampling another realization of this ensemble would measure statistically similar features. Then the time series can be assumed statistically stationary (homogeneous). Note that physical heterogeneity in space-time does not preclude statistical homogeneity-stationarity in the sense that the moments (e.g., mean and variance) are unaffected by the choice of space-time origin. We further assume that a typical clump size is both much larger than the elementary counting volume (time interval) and much smaller than the total volume sampled (total observation time).

The Poisson distribution is a function of one parameter, the mean value (equal to the variance). There are two natural generalizations of the Poisson process in the statistical literature: 1) a Poisson process having a time-dependent mean, and 2) a Poisson process with the mean as a random variable (the so-called doubly stochastic Poisson process or the "Poisson mixture," for short).

In the first, the mean is viewed as a time-varying but deterministic variable. As a result, the distribution for the number of drops observed during time interval $(0$, $T$ ) is given by (see Ochi 1990, 437-439)

$$
p(k)=\frac{\left(\int_{0}^{T} \bar{k}(t) d t\right)^{k}}{k !} \exp \left(-\int_{0}^{T} \bar{k}(t) d t\right) .
$$

It is important to note that this distribution still has the property that the mean and variance are equal.

In the second case of the Poisson mixture, however, $\bar{k}$ in (1) is no longer a constant but is itself a random (unpredictable) variable. Indeed, consider a given time series consisting of various rain patches each having a constant $\bar{k}$. Then the Poisson distribution applies to each patch as long as the elementary counting intervals are small, compared with the patch duration. Thus, the Poisson distribution must be considered a conditional one; that is, it is conditioned upon the fact that a random count is drawn from a patch having a mean value $\overline{\mathrm{k}}$. If the entire experiment duration involves several patches, the corresponding probability density functions (PDFs) must be added or "mixed." In other words, the $\bar{k}$ is itself an unpredictable and, therefore, a random variable. Hence, aside from the variance associated with a Poisson distribution, there is now an additional source of randomness introduced by the random variable $\bar{k}$. In order to derive an unconditioned PDF of the drop counts, we must then integrate over the probability distribution of the patches $\mathrm{f}(\bar{k})$ (e.g., Goodman 1985, 468474; Mandel et al. 1964, 449-450):

$$
p(k)=\int_{0}^{\infty} P(k \mid \bar{k}) f(\bar{k}) d \bar{k}=\int_{0}^{\infty} \frac{(\bar{k})^{k} \exp (-\bar{k})}{k !} f(\bar{k}) d \bar{k},
$$

where the vertical bar denotes conditional probability and $\bar{k}$ is the mean corresponding to each patch contributing to the mixture as described by the probability density function $\mathrm{f}(\bar{k})$. Moreover, it is well known that, for conditional random variables (e.g., Ochi 1990, 6566),

$$
\sigma_{k}^{2}=E_{\bar{k}}\left[\sigma^{2}(k \mid \bar{k})\right]+\sigma_{k}^{2}(E[k \mid \bar{k}]),
$$

where $E$ denotes the expectation value. Thus, the variance of a Poisson mixture is enhanced beyond that of a pure Poisson PDF by the variance of $\bar{k}$, that is, $\sigma_{k}^{2}=$ $\sigma_{P}^{2}+\sigma_{k}^{2}$ where the first term is the pure Poisson contribution, that is, $\sigma_{k}^{2}=\mu \equiv \int_{0}^{\infty} \bar{k} f(\bar{k}) d \bar{k}$, and $\mu$ is the global mean number of drops per unit volume ("global" here refers to long times compared with the coherence timescale).

The physical meaning is as follows. There are two sources of randomness: Poissonian fluctuations (represented by $\sigma_{P}^{2}$ ), which happen at a steady rain rate ( $\bar{k}=\mu$ held constant), and the rain rate fluctuations themselves $\sigma_{k}^{2}$. These sources of randomness are statistically independent and their variances therefore add. As an illustration of the effect of probability mixing, suppose $\mathrm{f}(\bar{k})$ is given by an exponential distribution of the form

$$
f(\bar{k})=\frac{1}{\mu} \exp \left(-\frac{\bar{k}}{\mu}\right) .
$$

This choice is often a good starting point because in the 
absence of any other information, this is the maximum entropy guess (the Boltzmann principle).

In passing, we note that in the case of monodisperse rain, (5) is equivalent to an exponential distribution of the mean rainfall rate in still air $\left(R_{0}\right)$ :

$$
R_{0}=0.6 \bar{k} \pi D^{3} V(D)
$$

where $\bar{k}$ in this context is the mean number of drops per cubic meter and $V(D)$ is the terminal fall speed $\left(\mathrm{m} \mathrm{s}^{-1}\right)$ of drops of diameter $D$. Because all the terms except $\bar{k}$ are constants in (6), an exponential distribution of $\bar{k}$ implies an exponential distribution of $R_{0}$.

With (5) inserted into (3) for $f(\bar{k})$, the integration can be readily performed to yield the geometric distribution

$$
p(k)=\frac{1}{\mu+1}\left(\frac{\mu}{\mu+1}\right)^{k},
$$

where $\mu$ is again the global ("long time") mean number of drops per unit volume.

The variance of the geometric distribution is (e.g., Goodman 1985)

$$
\sigma_{k}^{2}=\mu+\mu^{2},
$$

and scales as $\mu^{2}$ for large $\mu$, in sharp contrast to the Poisson distribution. In such cases, the relative fluctuation $\left(\sigma_{k} / \mu\right)$ no longer decreases with increasing sample mean, as opposed to the Poisson distribution. Instead, $\sigma_{k} / \mu$ approaches unity no matter how many drops are counted!

Other distributions are possible, of course. For example, if $\mathrm{f}(\bar{k})$ were a gamma distribution, $p(k)$ would then be described by the negative binomial distribution (Ochi 1990, 438-439). That is, if $\mathrm{f}(\bar{k})$ is given by

$$
f(\bar{k})=\frac{1}{\Gamma(m)} \lambda^{m} \bar{k}^{m-1} \exp (-\lambda \bar{k}) ; \quad \lambda \equiv \eta^{-1},
$$

with parameters $m$ and $\eta$, then (3) yields

$$
p(k)=\left(\begin{array}{c}
k+m-1 \\
k
\end{array}\right)\left(\frac{1}{1+\eta}\right)^{m}\left(\frac{\mu}{1+\eta}\right)^{k} ; \quad \eta=\frac{\mu}{m}
$$

where $\mu$ is the global average. For such a distribution, the parameters $\eta$ and $m$ can then be adjusted to fit the mean, $\mu$, and the variance to a simple relation:

$$
\sigma_{n}^{2}=\mu+\frac{\mu^{2}}{m} \text {. }
$$

There are several reasons that make this methodology convenient. First, the $m=1$ case reduces to the geometric distribution, while for very large $m,(10)$ reduces to the Poisson distribution (Ochi 1995, 105). Second, it has been used extensively to model bunching and clustering in other fields (Ochi 1995). Moreover, it has an appealing physical interpretation when the exponential distribution of clumps is applicable. Then, the $m=1$ case corresponds to $t \ll \tau$ regime

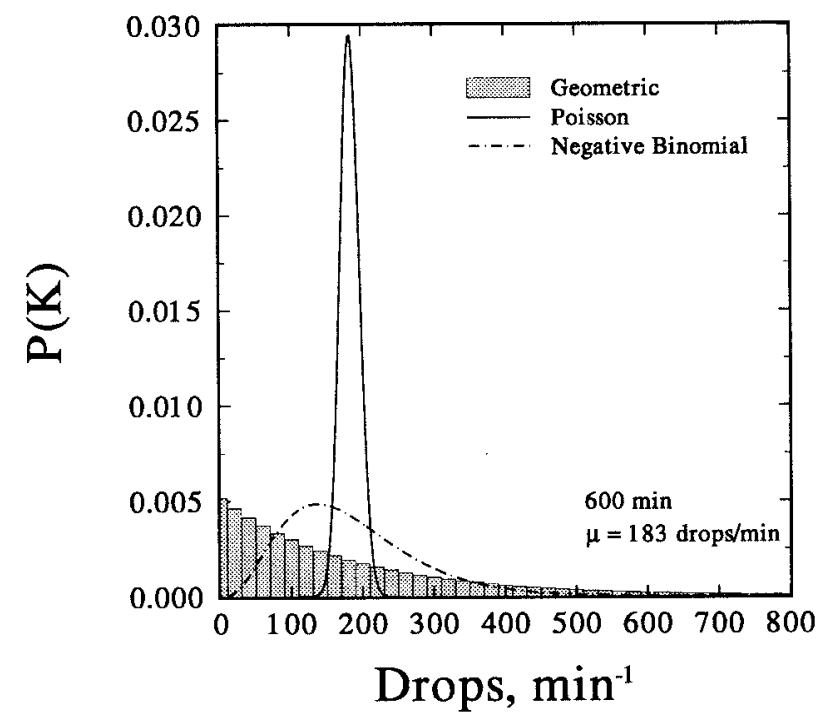

FIG. 2. The PDF of a Poisson distribution having $\mu=183 \mathrm{~min}^{-1}$ compared to the geometric histogram for a Poisson mixture having an exponential PDF of $\bar{k}$, that is, $f(\bar{k})=(1 / \mu) \exp (-\bar{k} / \mu)$ and the negative binomial distribution for a gamma PDF of $\bar{k}$ (see text) corresponding to the same mean but with a variance of $8555 \mathrm{~min}^{-2}$ and parameter $m=4$.

(the geometric distribution), while for very large $m$ and for very long counting intervals $(\tau \ll t), m$ may be interpreted as $t / \tau$ (number of coherence times in a counting interval).

As an example, Fig. 2 illustrates the effects of distribution mixing for the cases of an exponential $\mathrm{f}(\bar{k})$ for which a disdrometer measures a mean value $(\mu)$ of 183 drops per minute $\left(\mathrm{min}^{-1}\right)$ over a period of 600 min, and for a negative binomial distribution with the same mean and with $m=4$. Also included in the figure is the PDF corresponding to a pure Poisson distribution with the same mean value (i.e., corresponding to either stationary or a time-dependent Poisson process as discussed above). The variance of the mixtures is seen to be much larger than the Poissonian one. The increased variance is due to the patchiness (variability) of the rain, a feature completely hidden when computing only a mean value. For drops of one size, this figure also suggests that the increased variance and deviation from Poisson statistics should be striking and, therefore, readily detectable.

We conclude this section by describing one more tool, specifically designed to detect departures from the Poissonian behavior. For a general statistically stationary random process in time, the joint probability $P(1,2)$ of detecting two droplets in very short nonoverlapping time intervals of durations $\delta t_{1}$ and $\delta t_{2}$ is

$$
P(1,2)=(\bar{k})^{2}\left[\delta t_{1} \delta t_{2}+\eta(t)\right],
$$

where $\eta(t)$ is the excess two-point correlation function and $t$ is the lag time between the two intervals (e.g. Mandel et al. 1964). The $\eta(t)$ is defined as 


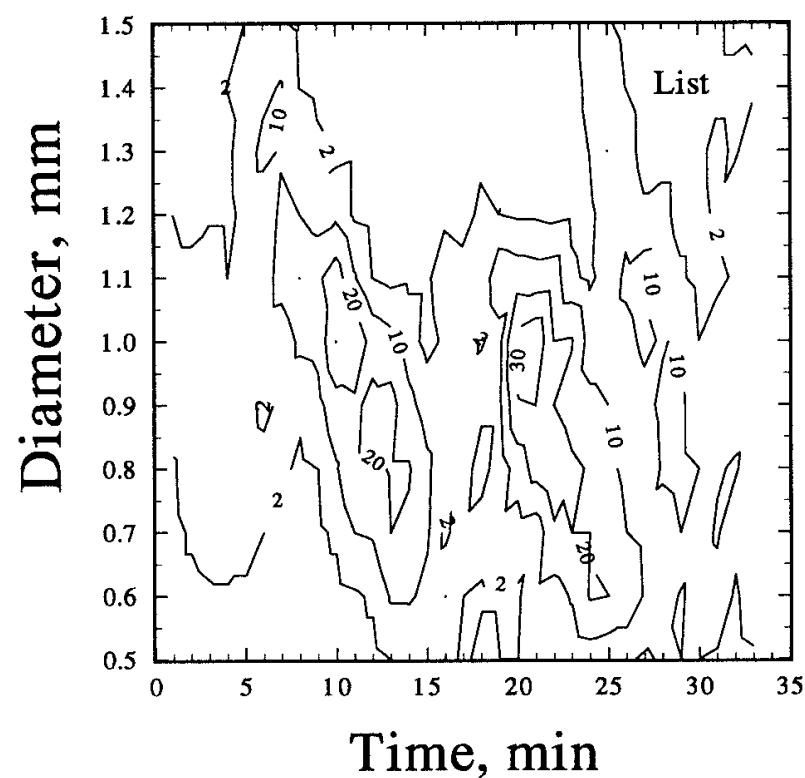

FIG. 3. Contour plot of the data of List et al. (1988) in light stratiform rain. The contours are in drops per minute as functions of drop size and time.

$$
\eta(t) \equiv \frac{\left[\overline{k(t) k(0)}-\mu^{2}\right]}{\mu^{2}}=\frac{\overline{k(t) k(0)}}{\mu^{2}}-1 .
$$

Note from (12) that the assumption of statistical independence of counts in nonoverlapping time intervals implies $\eta(t)=0$. Equivalently, from the definition of $\eta(t)$ in (13), it can be seen that the two-point autocorrelation function is zero in the absence of correlations (the Poissonian case). Hence, it is the violation of this statistical independence assumption and the presence of correlations that is characteristic of clumpiness of rain or bunching of raindrop arrival times. Consequently, clumpiness can be detected by measuring $\eta(t)$ directly for various delay times.

\section{Analyses of observations}

The definition of $\mathrm{f}(\bar{k})$ in (3) implies that over some temporal duration or spatial extent there is a definable characteristic "coherence time" (distance) to the measurements over which $\bar{k}$ may be considered approximately constant. We will use this coherence time as a measure of patch size. In order to estimate the $\bar{k}$ of a patch, the counting interval $t$ must be smaller than the coherence time $\tau$ of the patch of rain, so that many trials can be performed. On the other hand, the full description of $f(\bar{k})$ requires that the entire observation period $T$ be much larger than $\tau$. Hence, in order to estimate $f(\bar{k})$ with sufficient resolution and accuracy, we must satisfy

$$
t \ll \tau \ll T .
$$

The question then is how do such criteria apply to real measurements? It appears that under some circumstanc-

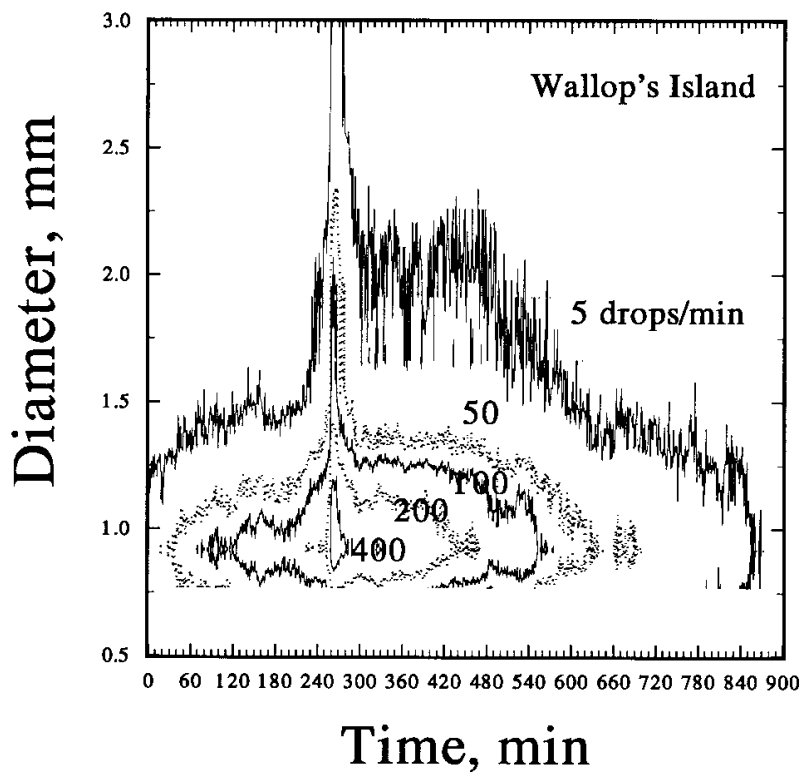

FIG. 4. Similar to Fig. 3 except for the data from the NASA Wallop's Island flight facility.

es, these criteria are met for data collected by a JossWaldvogel (JW) disdrometer (Joss and Waldvogel 1967). This instrument determines the size of drops as they impact a surface, places them in a size bin category, and counts the number in a bin over a period of time, typically $1 \mathrm{~min}$. In this work we consider two sets of data, one being the passage of a squall line over Wallop's Island, Virginia, and a second being measurements by List el al. (1988) in "streaky," light stratiform rain.

These data are illustrated in Figs. 3 and 4 as contour plots of the number of drops per minute as a function of time and drop size. Figure 3 represents the data of List et al. (1988) collected over a 33-min period during ". . . intermittent, streaky warm/cold rain from a multiple stratiform deck. ..." Figure 4 represents a much more extensive set of data (almost 900 min or $15 \mathrm{~h}$ ) collected during the passage of a squall line (at about $240 \mathrm{~min}$ ) across the National Aeronautics and Space Administration (NASA) flight facility at Wallop's Island, Virginia, in June 1996. Most of the rain event appears to be due to weakly convective stratiform clouds associated with the squall. In Fig. 3 there is clear evidence of patches having timescales $(\tau)$ on the order of 2-3 min. In Fig. 4, the fluctuations in the contours also suggest fine-scale structures.

In order to define more objectively a timescale for the patches, we calculate the normalized autocovariance function (Fig. 5a) of the time series of 1-min counts for different drop sizes during the period of more intense rain from about 240 to $500 \mathrm{~min}$ (see Fig. 5). The normalized autocovariance is defined as

$\operatorname{cov}[k(t), k(t+L)]=\frac{\left[\overline{k(t) k(t+L)}-\mu^{2}\right]}{\overline{k^{2}}-\mu^{2}}$, 

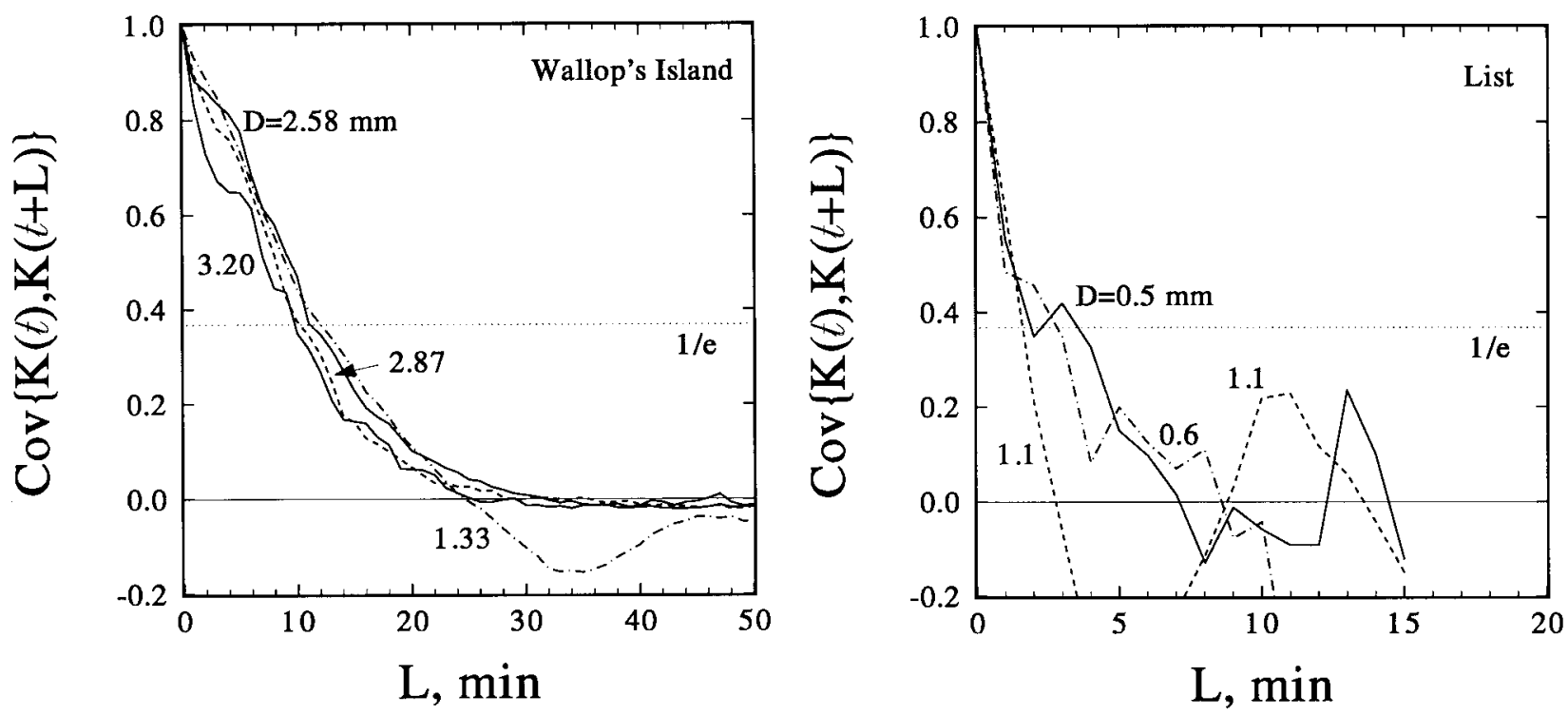

FIG. 5. $\operatorname{Cov}\left\{(K(t), K(t+\mathrm{L})\}=\left\{<K(t) K(t+\mathrm{L})>-<K>^{2}\right\} /\left\{<K^{2}(t)>-<K>^{2}\right\}\right.$ for lags $L$ and for (a) the Wallop's Island data for four different drop sizes. There is a coherence time, $\tau \approx 10 \mathrm{~min}$, for these data. (b) For List's data, $\tau \approx 2 \mathrm{~min}$.

where $L$ is the time lag. From sizes of 1.33 up to 3.2 $\mathrm{mm}$ diameter, the autocovariance drops to $(1 / e)$ at about $10 \mathrm{~min}$ for the Wallop's Island data. Consequently, we choose that time as the temporal patch size. Since $t=$ $1 \mathrm{~min}, \tau=10 \mathrm{~min}$, while $T=900 \mathrm{~min}$, the Poisson mixture criteria (14) are satisfied. On the other hand, for List's data (Fig. $5 \mathrm{~b}$ ), $\tau$ is about $2 \mathrm{~min}$, so that $\tau, t$, and $T$ only partially meet the criteria (14).

The effect of rain clumpiness is evident in the histograms of minute by minute counts as illustrated in Fig. 6 where Poisson distributions corresponding to each mean $(\mu)$ are also plotted. Clearly, in all three examples from the Wallop's Island data, the observed distributions are considerably broader (by factors of 10-100 in some cases) than would be the case if the statistics were those of a Poisson process. In other words, it appears that

1) the statistics are not consistent with either a stationary or a time-dependent Poisson process;

2) the results may perhaps be described by a Poisson mixture (because the geometric PDFs fit the data reasonably well in spite of using no adjustable parameters); and

3) the variance is dominated by the rain variability (variance of $\bar{k}$ ).

Although it is impossible to prove the validity of the second point rigorously, we can test the consistency of the argument by constructing Poisson mixture approximations to some data using $\tau=10 \mathrm{~min}$ as a definition of patch size. Two examples are given of this consistency test. In the first, we consider a 262-min period at the time of the most intense rain (see Fig. 4). We take 26 10-min averages to estimate the mean number of counts, which are then used to define $\mathrm{f}(\bar{k})$ in (3). The
Poisson mixture is calculated next and plotted along with the "equivalent" Poisson distribution (defined as Monte Carlo simulated time series having the observed mean value during that period). The results are illustrated in Fig. 7. The Poisson mixture essentially reproduces the observed mean, explaining about $85 \%$ of the observed variance, while the Poisson PDF captures only about $4 \%$ of the variance. If we then consider the entire $900 \mathrm{~min}$ of measurements and repeat the process, the Poisson mixture closely reproduces the observed distribution (Fig. 8), capturing about $93 \%$ of the variance. On the other hand, the Poisson PDF (stationary or timedependent) is clearly inadequate.

With regard to List's data, Fig. 9 also suggests that the Poisson distribution having the observed mean does not fit these data well. On the other hand, the geometric distribution, having the observed mean (like the Poisson distribution) and using no additional information, appears to fit the data relatively well. Unfortunately, $t$ and $\tau$ are comparable and it is not possible to determine $f(\bar{k})$ with sufficient clarity to calculate a meaningful Poisson mixture approximation. (This example demonstrates the need for finer temporal resolution in such disdrometer measurements.)

However, this is not to say that the Poissonian approach is always invalid for the 1-min time series. Figure 10a illustrates the time series of counts corresponding to 1.66 -mm drops over a 180 -min period of unusually steady drop flux during the postconvective stratiform rain (Wallop's Island data). The observations at the top are almost equal to an equivalent Poisson distribution, that is, to a random number-generated Poisson distributed time series with the same mean as that of the data $\left(10.03 \mathrm{~min}^{-1}\right)$. This is consistent with the near 

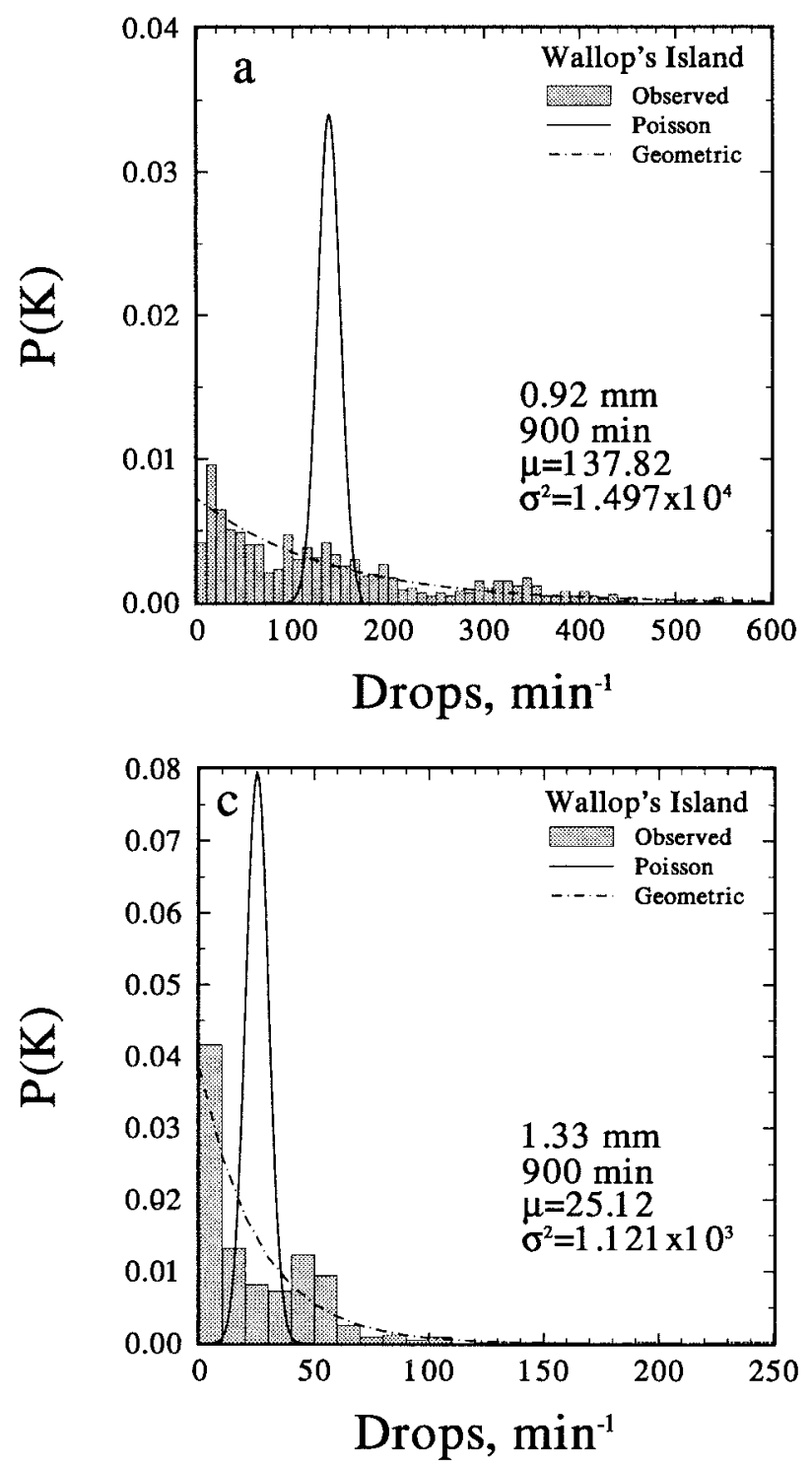

equality between the observed mean and variance (11.61). The "near" Poisson nature of the observations at this one size during this interval is also apparent in the similarity between the data histogram and the equivalent Poisson distribution (Fig. 10b). It is important to note, however, that correlations on a scale of, say, 10 s, are "washed out" in such 1-min time series and are difficult to detect without having finer temporal resolution.

This analysis is further confirmed by the calculation of the excess two-point autocorrelation function $\eta(t)$ presented in Fig. 11 and defined in (13). The "theoretical" value is simply meant to illustrate that $\eta(t)$ is identically zero in the Poissonian case. As anticipated in Fig. 10, $\eta(t)$ is seen to be nearly identically zero for the $1.66-\mathrm{mm}$-size data. On the other hand, the $1.33-\mathrm{mm}$ size data are consistent with the non-Poissonian char-

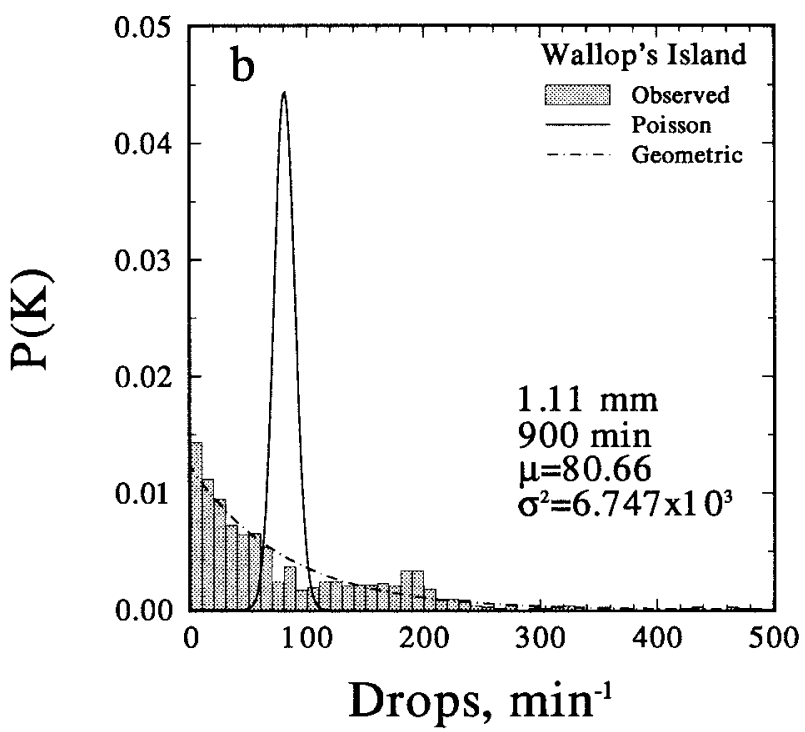

FIG. 6. The observed histograms of the number of drops per minute at three different drop sizes at Wallop's Island having the indicated means $\mu$ and variances $\sigma^{2}$. Poisson PDFs having the observed means (and corresponding to a time-dependent Poisson process) and geometric PDFs are also plotted for comparison. In all cases, the observed distributions are considerably broader than would be expected for time-dependent Poisson processes.

acter of the data in Fig. 6c. The non-Poissonian "bunching" of drops is most apparent for the larger 1.91-mm size where correlations are not only the strongest but also occur on a shorter timescale, as expected for convective storms.

The transition from the Poisson mixture to a pure Poisson distribution is explored in Figs. 12a-c. The 1.11-mm-size histograms are compared using the entire 900,300 , and $50 \mathrm{~min}$ (the latter intervals, being centered on the 445 th minute, do not include the squall line). The pattern illustrates the transition from the geometric to the Poissonian distribution as the experiment duration $(T)$ decreases. This pattern is well characterized by the changing shape of the negative binomial distribution of (10) as the parameter $m$ increases from 1 (for the geometric distribution) to a large value of 70 (recall that the Poissonian ap- 


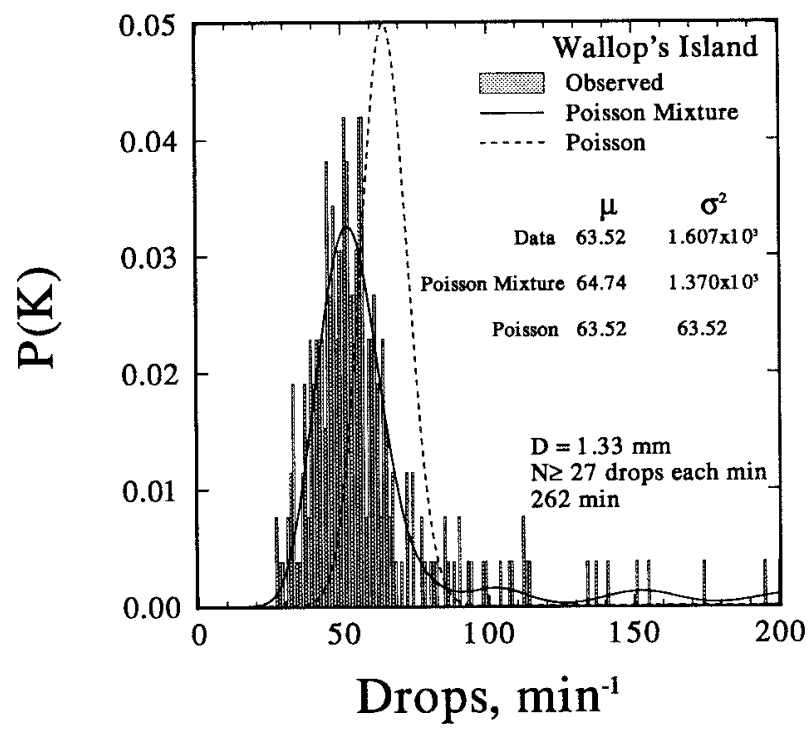

FIG. 7. A comparison of the observed histogram of the number of drops per minute and the PDFs expected for Poisson (dashed line) and Poisson mixture (solid line) processes at a drop size of $1.33 \mathrm{~mm}$ during the 262-min period of intense rain when the number of drops $(N)$ per minute $\geq 27$. The Poison mixture of 26 Poisson distributions (corresponding to $\tau=10 \mathrm{~min}$ ) is in good agreement with observations.

proximation is recovered in the limit of very large $\mathrm{m})$. The value of $\mathrm{m}$ was chosen to fit the observed variance and mean according to (11).

\section{Concluding remarks}

The results of this case study indicate that the effect of the variability (patchiness) of rain on drop sampling appears to be best described in terms of a Poisson mixture, at least on the timescales of these data. ${ }^{4}$ Such a description, in our opinion, is preferred to the timedependent Poisson description that yields results analogous to those of Cornford (1967).

In particular, a time series of counts of drops around $1.33 \mathrm{~mm}$ diameter is shown in Fig. 13. Using a locally weighted linear regression fit, we then compute a smooth "mean" curve to the data (heavy solid line in Fig. 13) and subtract this mean curve from the data to yield a time series of residual fluctuations. Cal-

\footnotetext{
${ }^{4}$ Our approach was inspired by the "light bucket"-antenna analogy (. . . to collect the light from the star like rain in a bucket and pour it onto the detector." (see Hanbury Brown 1974, 5), and by the Poisson mixture derivation of the Bose-Einstein (geometric) distribution by Mandel and et al. (1964). There, the mean light intensity is analogous to the mean rain rate, and raindrops arriving at a disdrometer are similar to photons arriving at a photodetector. Our clumpiness of rain is then analogous to the famous wave noise of Einstein, that is, mean intensity fluctuations of the blackbody radiation caused by random interference (similar to Rayleigh fading of radar echoes).
}

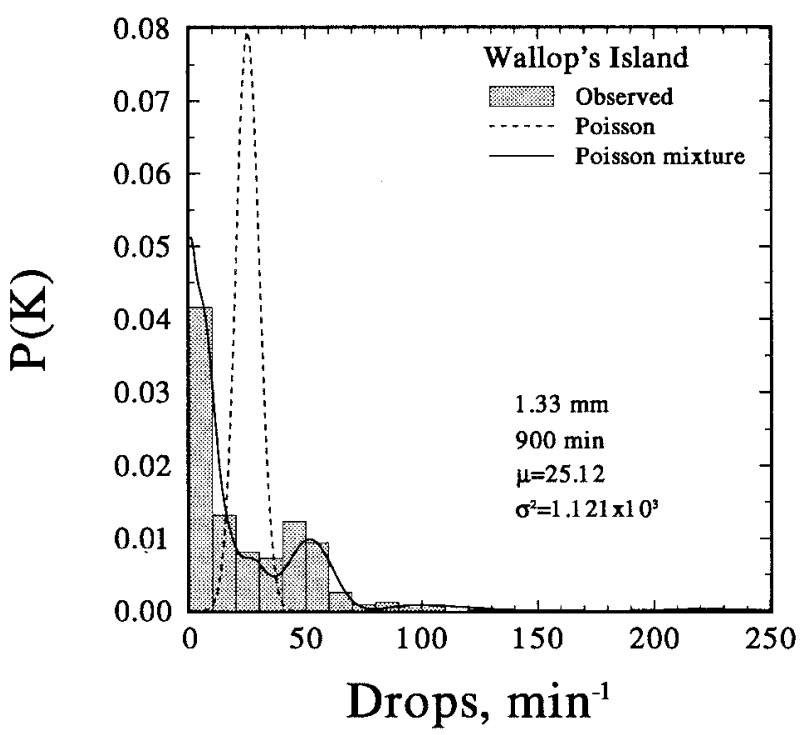

Fig. 8. Similar to Fig. 7 except that these plots correspond to the entire $900 \mathrm{~min}$ of Wallop's Island data and the Poisson mixture is a combination of 90 Poisson distributions (corresponding to $\tau=10$ min.).

culations show that the mean values of the data and mean curve are nearly identical (28.17 vs 28.23 $\min ^{-1}$, respectively), while that for the fluctuations is near zero $\left(-0.06 \mathrm{~min}^{-1}\right)$. Moreover, calculations show that the fluctuations are uncorrelated. The total variance of the data $\left(449.4 \mathrm{~min}^{-2}\right)$ is nearly equal (to

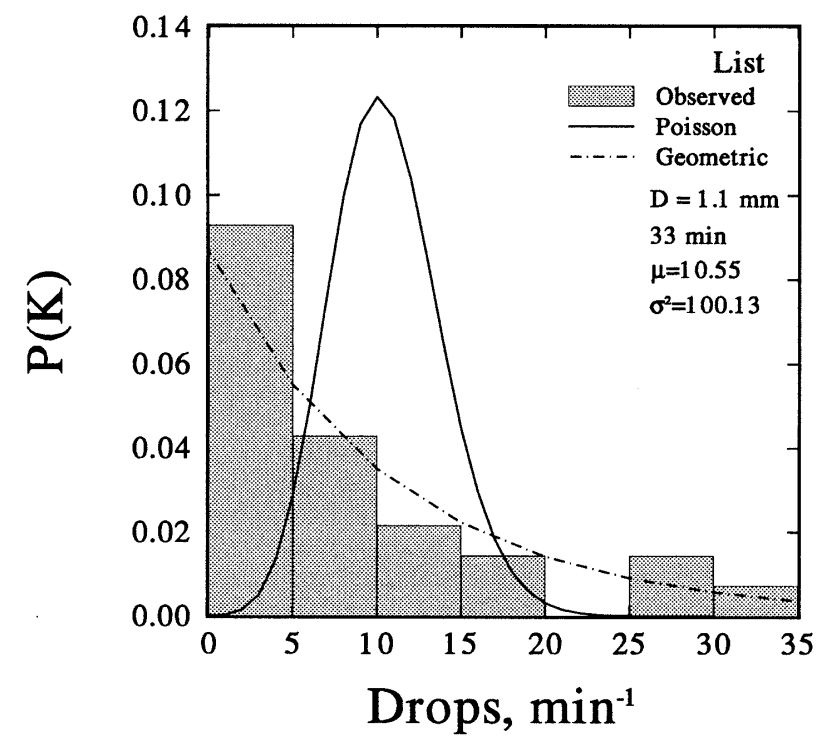

FIG. 9. A comparison between the observed histogram and the PDF expected for the time-dependent Poisson process (solid line) derived using List's data. The geometric PDF (dashed-dot line) based solely on the mean value provides a better approximation to these data. It was not possible to construct a Poisson mixture because the coherence time $\tau=2 \mathrm{~min}$ is too close to the sample time $t$. 

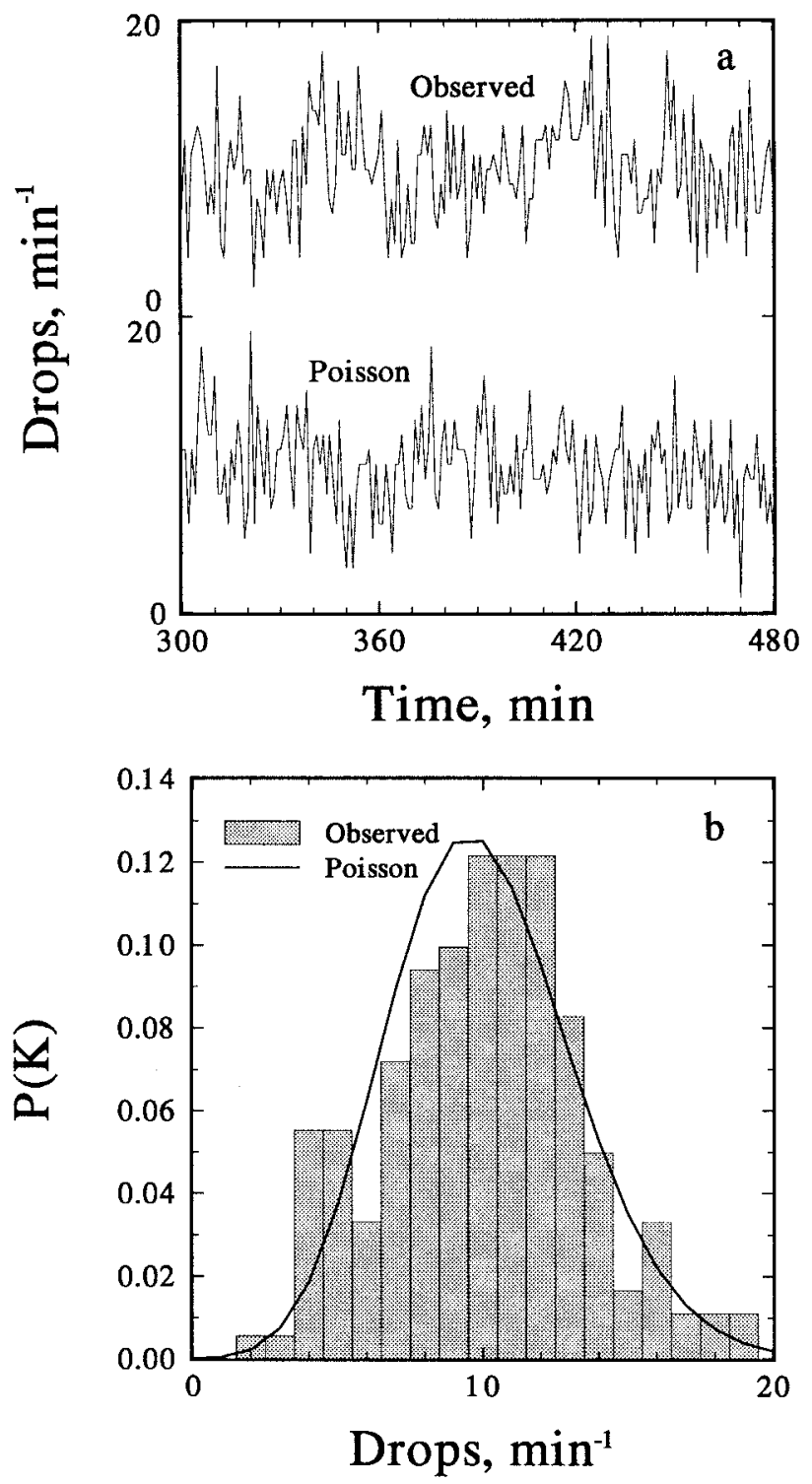

FIG. 10. (a) Time series of counts of 1.66-mm drops compared to an equivalent (i.e., the same mean value) Poisson time series during a time of unusually constant flux. (b) The histogram of the time series observations compared to the equivalent Poisson distribution.

within $99 \%$ ) to the sum of the variance of the mean fit $\left(410.9 \mathrm{~min}^{-2}\right)$ and that for the residual fluctuations $\left(34.6 \mathrm{~min}^{-2}\right)$. In addition, this latter variance is very near the mean, in agreement with (4). Thus, it appears that (3) is essentially satisfied, consistent with the Poisson mixture hypothesis. We must emphasize that simply because one can derive a mean curve by postprocessing the data does not imply that the original data statistics are then those of a time-dependent Poisson process. Indeed, at the time of experiments, the local mean variation is unpredictable and must, there-

\section{Evidence of Raindrop Bunching}

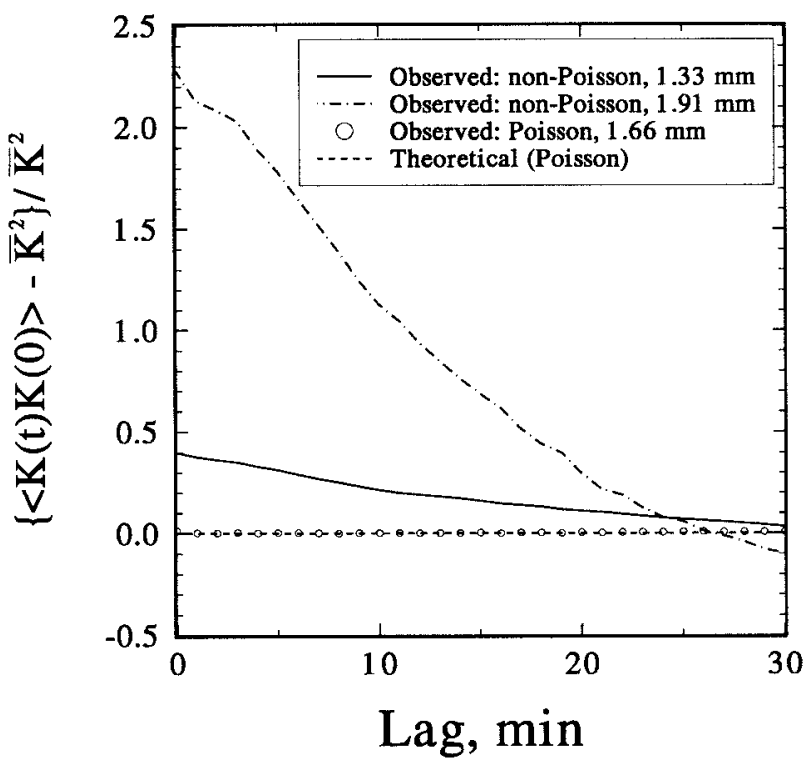

FIG. 11. Plots of the excess two-point autocorrelation function (see text) during the passage of a squall line for two different sizes of drops. The open circles correspond to 1.66- $\mathrm{mm}$ size drops (see Fig. 10) during a period of unusually steady flux after passage of the squall line.

fore, be viewed as an additional source of randomness.

What this work shows, then, is that given a time series of drop counts, the best strategy for estimating the mean is not always the standard (Cornford) approach of adding up samples. As Fig. 13 illustrates, the uncertainty in the estimated mean may actually increase as the sample size is increased when local conditions are highly variable. In such situations it may be beneficial to identify appropriate coherence scales based on a time-series methodology.

In the Wallop's Island data analysis we assumed (based on the data autocovariance functions) that the 1-min measurements over a 10-min period on average are all drawn from a Poisson distribution having an approximately constant mean. It should be remembered, however, that the convective, stratiform rain sampled here may have had unusually long autocovariance times and that the 1-min samples may have somehow masked some of the true variability. After all, fluctuations on scales finer than 10 min are evident in Fig. 1. In this analysis we attributed many of these fluctuations to random sampling from a Poisson distribution having a characteristic time scale of about $10 \mathrm{~min}$. Yet when looking at the data of List (Fig. 4) and at enlarged displays of the squall line data (Fig. 14), there are strong suggestions of structures on the scale of 2-4 min, consistent with List's data and very near the time resolution limit of disdrometers. With improved resolution (several seconds instead of min- 

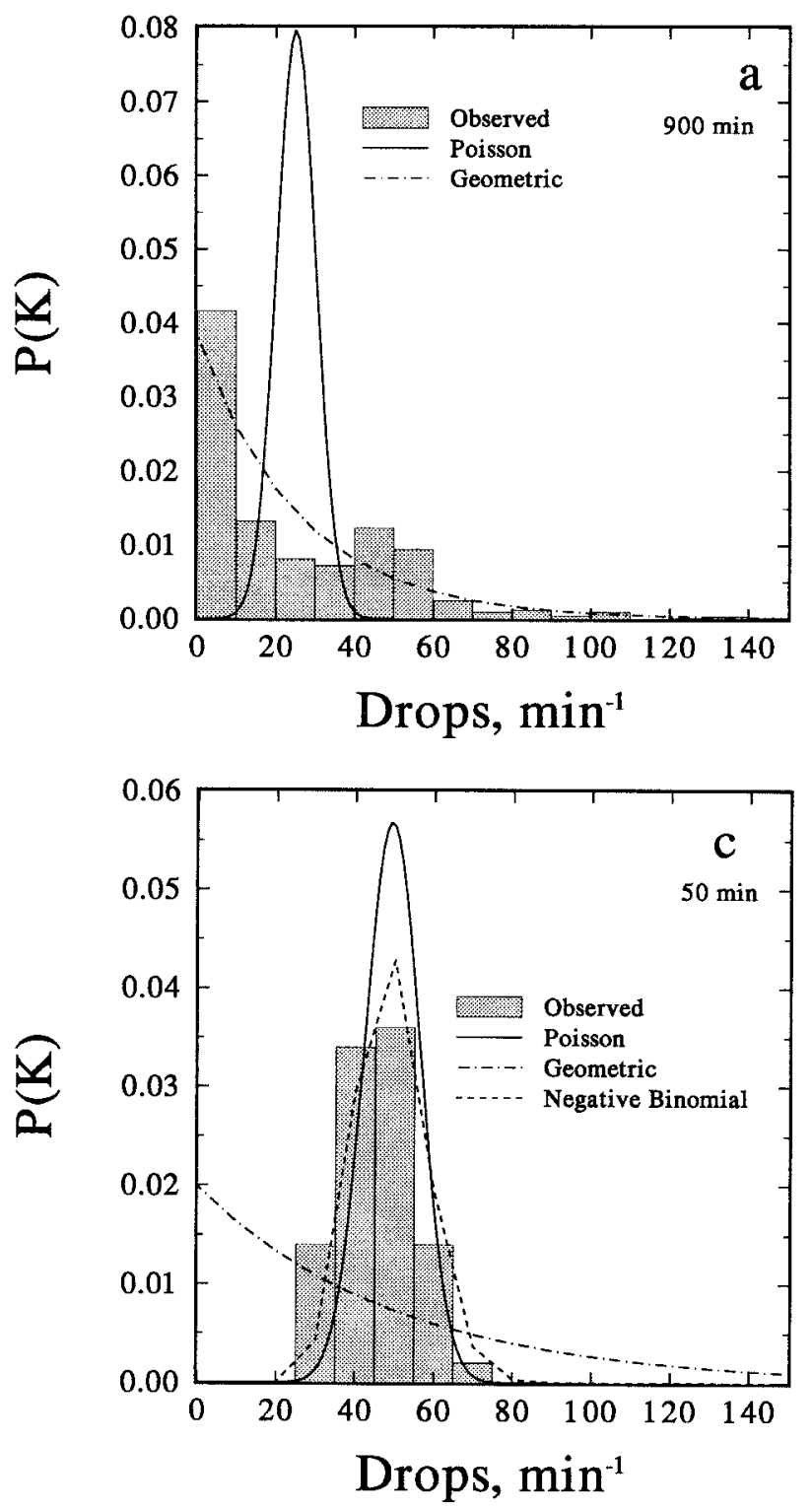

utes), it is not unreasonable to speculate that a considerable reduction might occur in the characteristic autocovariance times (e.g., from several minutes to several seconds). How would such fine structures influence the sampling strategy for determining mean drop concentrations and drop size distributions?

This analysis shows the importance of rain variability on sampling. Increased variability, whether experienced by an aircraft flying through both stratiform and convective cores during sampling or by disdrometers as complex storms pass overhead, obviously leads to increased uncertainty in the interpretation of a mean. At the bottom of Fig. 8, for example, the 900-min mean value is about 25 drops per minute, but the standard deviation of the distribution is still 33 drops per minute (even after $900 \mathrm{~min}$ ). As the variance expressions (8)

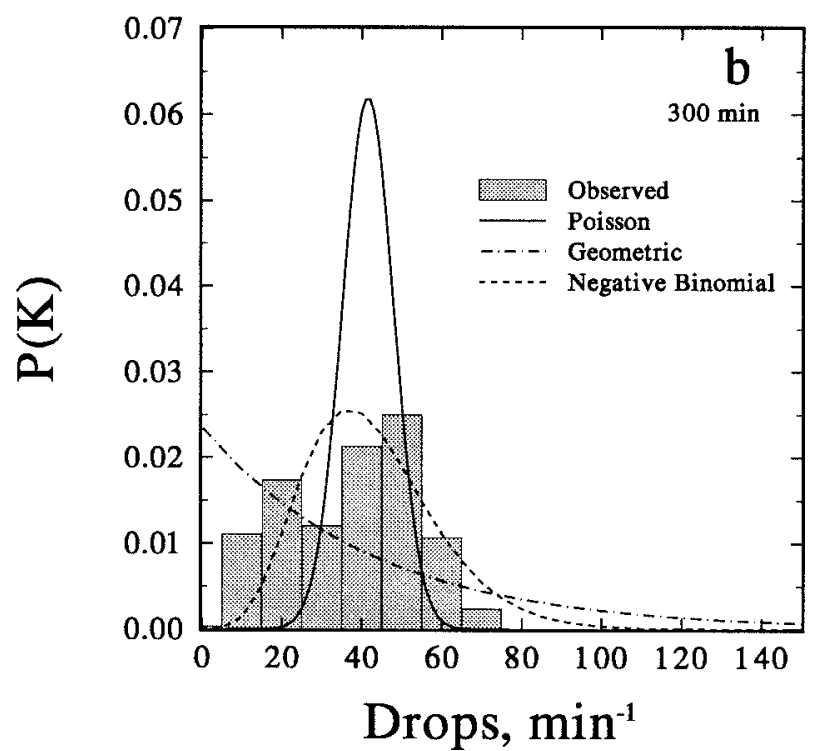

Fig. 12. Histograms of counts of 1.11-mm drops as functions of the length of sampling time ( $T$ ) compared to equivalent (i.e., the same mean value) fits for Poisson, geometric, and negative binomial pdfs. Note that as $T$ decreases the histograms undergo a transition from (a) nearly geometric for $900 \mathrm{~min}$, (b) to approximately negative binomial $(m=3)$ for $300 \mathrm{~min}$, and (c) to nearly Poisson for $50 \mathrm{~min}$. Here, $m=70$ for the negative binomial in (c).

and (11) show, the traditionally assumed $1 / \sqrt{N}$ dependence of the relative sampling error on the total number of drops $(N)$ cannot be safely assumed when conditions are changing rapidly.

The very meaning of a drop size distribution becomes sensitive to the counting duration in such cases. For instance, if long times are required to collect an adequate sample of large drops, what then happens to the uncertainty associated with the mean of smaller drops? It appears likely that the Poissonian treatment of raindrop sampling statistics implied by Cornford will not always apply, and that the appealing and simple sampling criteria requiring 23 drops per bin size may, at times, not only miss the importance of the effect of variability on the measurements but may actually lead to increased uncertainty in the mea- 


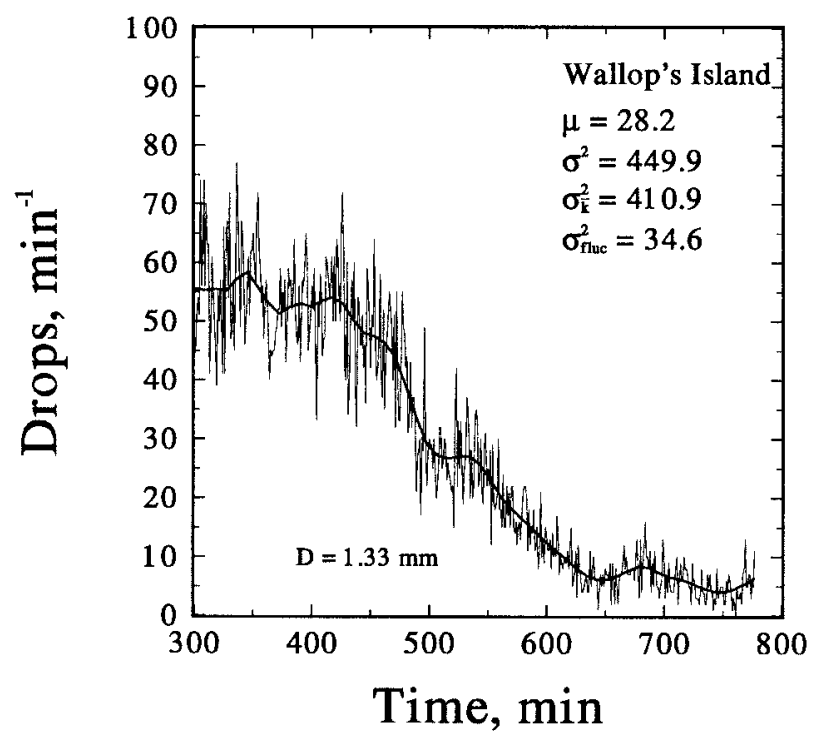

FIG. 13. Joss-Waldrogel disdrometer time series data corresponding to a mean drop diameter of $1.33 \mathrm{~mm}$ with an overlay of a least square error fit (solid line). Here $\mu$ is the mean number of drops per minute over all the data, $\sigma^{2}$ is the variance of the observed data, $\sigma_{k}^{2}$ is the variance of the mean fit, and $\sigma_{\mathrm{fw}}^{2}$ is the variance of the residual fluctuations (very close to $\mu$ ). The fact that $\sigma^{2}$ is very nearly equal to the sum of $\sigma_{k}^{2}$ and $\sigma_{\text {fluc }}^{2}$ suggests that the Poisson mixture model is applicable in this instance.

surements of other smaller drops. This is explored further in Jameson and Kostinski (1997).

From the meteorological perspective, raindrop bunching can be visualized in several ways. For example, one patch at one height might pass over patches at lower altitudes. In addition, patches may overlap because of, say, differential advection among clusters at different altitudes in the presence of vertical wind shear or when there are horizontal gradients in the vertical wind shear. Consequently, when one imagines overlapping patches particularly across spectrum of sizes, it is not hard to visualize the possibility of a real, clumpy mixture of the actual drops. ${ }^{5}$

However, at this stage, one must regard such suggestions as speculative. Anyone who has seen the one or two second "waves" of drop concentrations apparent either as visible attenuation aloft or as increased splashing on the pavement during a thunderstorm knows that the detection of such features probably requires a counting resolution on the order of seconds rather than the minutes of these data. In lieu of such high-resolution

\footnotetext{
${ }^{5}$ An intriguing possibility is that the bunching associated with deviations from the Poisson statistics may also lead to nonexponential interarrival (waiting) times. Indeed, the exponential probability distribution of waiting times between consecutive drop arrivals is but another characterization of a stationary Poisson process (e.g., Ochi 1990). Bunching of drops causes departures from the exponential distribution that should be detectable when arrival times are recorded.
}

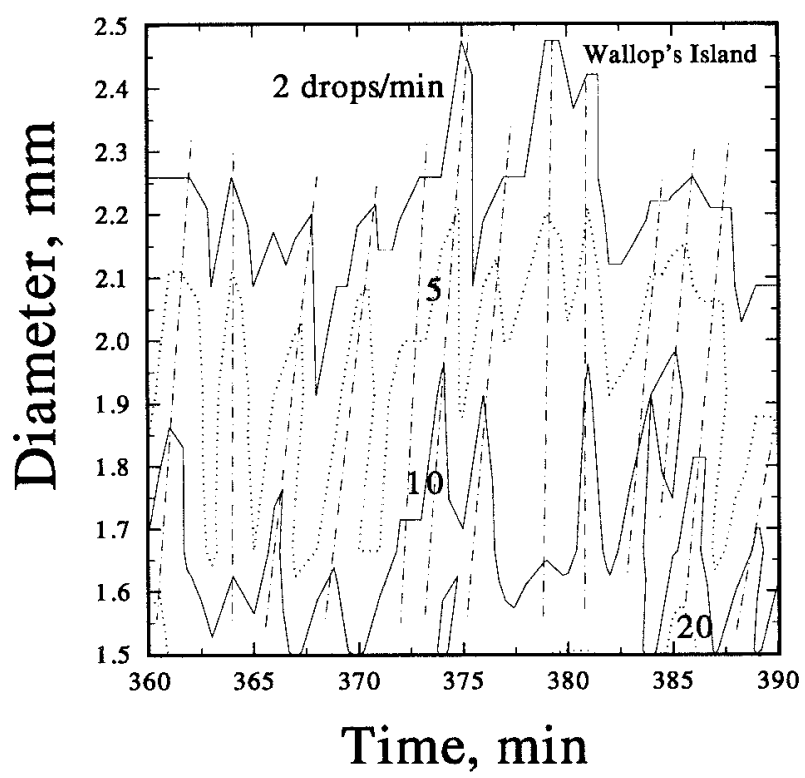

Fig. 14. Contour plot of $30 \mathrm{~min}$ of data from Wallop's Island showing what are apparently 2-3-min fine-scale structures similar to those found in List's data. The dashed lines are intended to highlight apparent approximately 3-min structures (impulses) in the observations.

disdrometer measurements, one might anticipate that physical clustering of raindrops would alter their correlation functions or the arrival times, causing readily detectable deviations from those anticipated for a Poisson distribution.

Acknowledgments. This work was supported under grants from the National Science Foundation [ATM95-12685 (AK) and ATM-9419523 (AJ)] with additional funding from the National Aeronautics and Space Administration under NAS5-32780 (AJ). We thank Mr. Otto Thiele of NASA (Goddard Space Flight Center) and Mr. Gene Furness of NASA (Wallop's Island facility) for providing the essential disdrometer data from Wallop's Island.

\section{REFERENCES}

Bayewitz, M. H., J. Yerushalmi, S. Katz, and R. Shinnar, 1974: The extent of correlations in stochastic coalescence process. J. Atmos. Sci., 31, 1604-1614.

Cornford, G. S., 1967: Sampling errors in measurements of raindrop and cloud droplet concentration. Meteor. Mag., 96, 271282.

Crane, R. K., 1990: Space-time structure of rain rate fields. J. Geophys. Res., 95, 2011-2020.

Feller, W., 1968: An Introduction to Probability Theory and Its Applications. Vol. 1, John Wiley and Sons, 478 pp.

Gillespie, D. N., 1972: The stochastic coalescence model for cloud droplet growth. J. Atmos. Sci., 29, 1496-1510.

_ 1975: Three models for the coalescence growth of cloud drops. J. Atmos. Sci., 32, 600-607.

Goodman, J. W., 1985: Statistical Optics. John Wiley and Sons, 500 pp. 
Hanbury Brown, R., 1974: The Intensity Interferometer. Taylor and Francis Publishers, $281 \mathrm{pp}$.

Jameson, A. R., and A. B. Kostinski, 1997: Fluctuation properties of precipitation. Part II: Reconsideration of the meaning and measurement of raindrop size distributions. J. Atmos. Sci., 54, in press.

Johnson, D. B., 1982: The role of giant and ultragiant aerosol particles in warm-rain initiation. J. Atmos. Sci., 39, 448-460.

Joss, J., and A. Waldvogel, 1967: Ein Spektrograph fur Niederschlagstropfen mit automatisher Auswertung. Pure Appl. Geophys., 68, 240-246.

List, R., D. Hudak, R. Nissen, N. P. Tung, S. K. Soo, and T. S. Kang, 1988: Raindrop size distributions in warm rain at $\mathrm{Pe}$ nang. Tropical Rainfall Measurements, J. S. Theon and N. Fugono, Eds., Deepak Publishing, 271-278.

Mandel, L., E. C. D. Sudarshan, and E. Wolf, 1964: Theory of pho- toelectric detection of light fluctuations. Proc. Phys. Soc., 84, $435-444$.

Ochi, M., 1990: Applied Probability and Stochastic Processes. John Wiley and Sons, $499 \mathrm{pp}$.

Pruppacher, H. R., and J. D. Klett, 1978: Microphysics of Clouds and Precipitation. Reidel, 714 pp.

Rogers, R. R., and M. K. Yau, 1989: A Short Course in Cloud Physics. Pergamon Press, 229 pp.

Twomey, S., 1964: Statistical effects in the evolution of a distribution of cloud droplets by coalescence. J. Atmos. Sci., 21, 553-557.

van Kampen, N. G., 1992: Stochastic Processes in Physics and Chemistry. North-Holland Press, $465 \mathrm{pp}$.

Wilks, D. S., 1995: Statistical Methods in the Atmospheric Sciences. Academic Press, $467 \mathrm{pp}$.

Young, K. C., 1993: Microphysical Processes in Clouds. Oxford University Press, $427 \mathrm{pp}$. 